\title{
Synthesis and Theoretical Modeling of Crystalline Pb Nanowires.
}

\author{
P.Santiago, ${ }^{*}$ M. Peréz-Alvarez,** , and L. Rendón* \\ * Instituto de Física, Universidad Nacional Autónoma de México, A.P. 20-364, 01000 México D.F. \\ ** Instituto Nacional de Investigaciones Nucleares K.m. 36.5, Salazar, Edo. de Mexico
}

Nanowires are one of the 1-dimension nanostructures studied in nanoscience and nanotechnology for their potential application in nanoelectronic devices, optical and chemical sensors, high density data store devices and many other applications. This interest has been increased in the last few years and new methods of synthesis have been developed. One of the most controllable and inexpensive methods to produce one-dimensional nanostructured materials is the use of templates, such as $\mathrm{Al}_{2} \mathrm{O}_{3}$ nanoporous molds prepared by anodization (AANTs) process with channels and pore density controlled by the electrolytic cell conditions. On the other hand, the template method itself generates the synthesis of one-dimension nanostructure arrays supported into an insulated matrix with high thermal and chemical stability. Therefore, the AANTs filled with any material can be used as a nonodevice itself.

In this work we report the synthesis of lead nanowires synthesized by thermal decomposition of lead acetate into AANTs with an average porous inner-diameter of $20 \mathrm{~nm}$ [1]. We used $0.1 \mathrm{M}$ solution of lead acetate in dimethylformamide (DMF) as solution phase precursor. The AANT was immersed in the precursor solution for $5 \mathrm{~min}$ and then dried at $70^{\circ} \mathrm{C}$ to evaporate the remaining solvent. The precursor was pyrolized at $450^{\circ} \mathrm{C}$ for $1 \mathrm{~h}$ inside a programmable horizontal furnace under the flow of hydrogen and nitrogen gas mixture $\left(10 \% \mathrm{H}_{2}, 20 \mathrm{~mL} / \mathrm{min}\right)$. The structural characterization was performed in a JEM 2010 FasTem microscope equipped with a Noran EDS spectrometer. The sample was prepared for its observation using a mortar in order to crack the alumina mold and liberates the nanowires without any further chemical process.

High resolution images are present in figure 3 . We can observe single crystal nanowires with length around of $10 \mu \mathrm{m}$ and an average diameter of 15 to $20 \mathrm{~nm}$. However, some of the wires present multiple twining planes showing a polycrystalline structure. The EDS obtained in nanobeam mode $(0.5 \mathrm{~nm}$ spot size) shows that the chemical composition of the NW is fundamentally $\mathrm{Pb}$. The wires are growing principally in [111] direction $(0.29 \mathrm{~nm})$.

Once we obtained the experimental result we used Montecarlo or Simulated Annealing algorithms to simulate one-dimension $\mathrm{Pb}$ nanostructures [2]. This method is based on an aleatory search of minimum structures. The approach to resolve the problem was to find the most stable configuration. Graphical view is shown in figure 1. We built an initial structure and then grew it in a specific direction for $\mathrm{Pb}$ element (figure2). We studied the nanowires evolution using Simulated Annealing based in a thermodynamic process. The Temperature parameter $(\mathrm{T})$ simulates the potential energy of the structure and system evolution goes to a minimum energy system $\left(T_{f}\right)$. The theoretical models show in figure 3 simulates the evolution of the nanowires during the thermal process. There is a perfect match between the experimental and theoretical results.

References

[1] C.Peza-San German, P. Santiago, et. al., J. Phys. Chem. B 109 (2005) 17488.

[2] R. Werner., Eur. Phys. J.B. 43, (2005) 47.

[3] We kindly acknowledge to Instituto de Física at UNAM for allowing the use of their microscopy facilities. This work is supported by a grand from CUDI-CONACyT. We also acknowledge the assistance of Mr. Angel Flores and Diego Quiterio in the sample preparation process. 


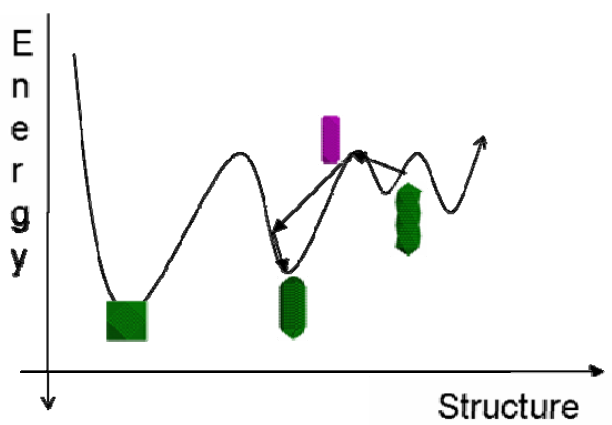

Figure 1. Schematic diagram shows the minimum energy for diverse 1-D structures as a function of the element and structures.

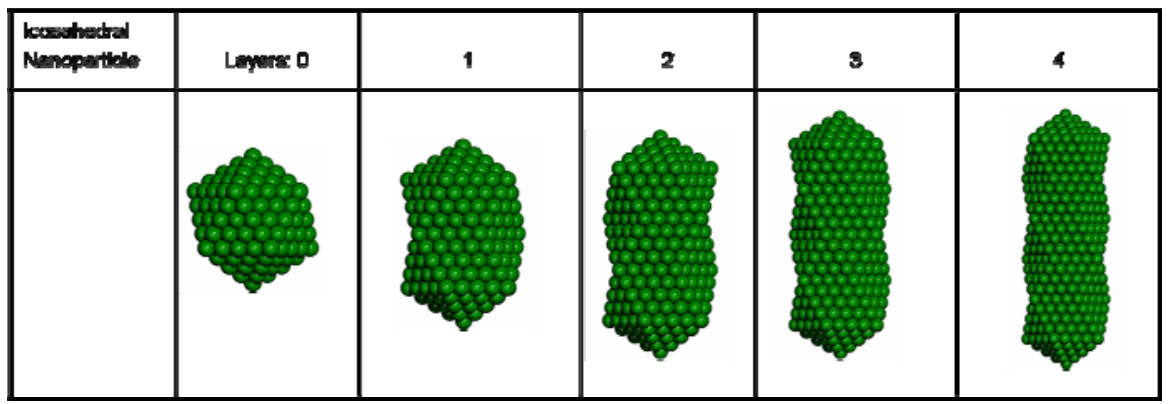

Figure 2. An initial structure is built (icosahedral nanoparticle) and then grown in a specific direction in order to assemble a 1-D texture.
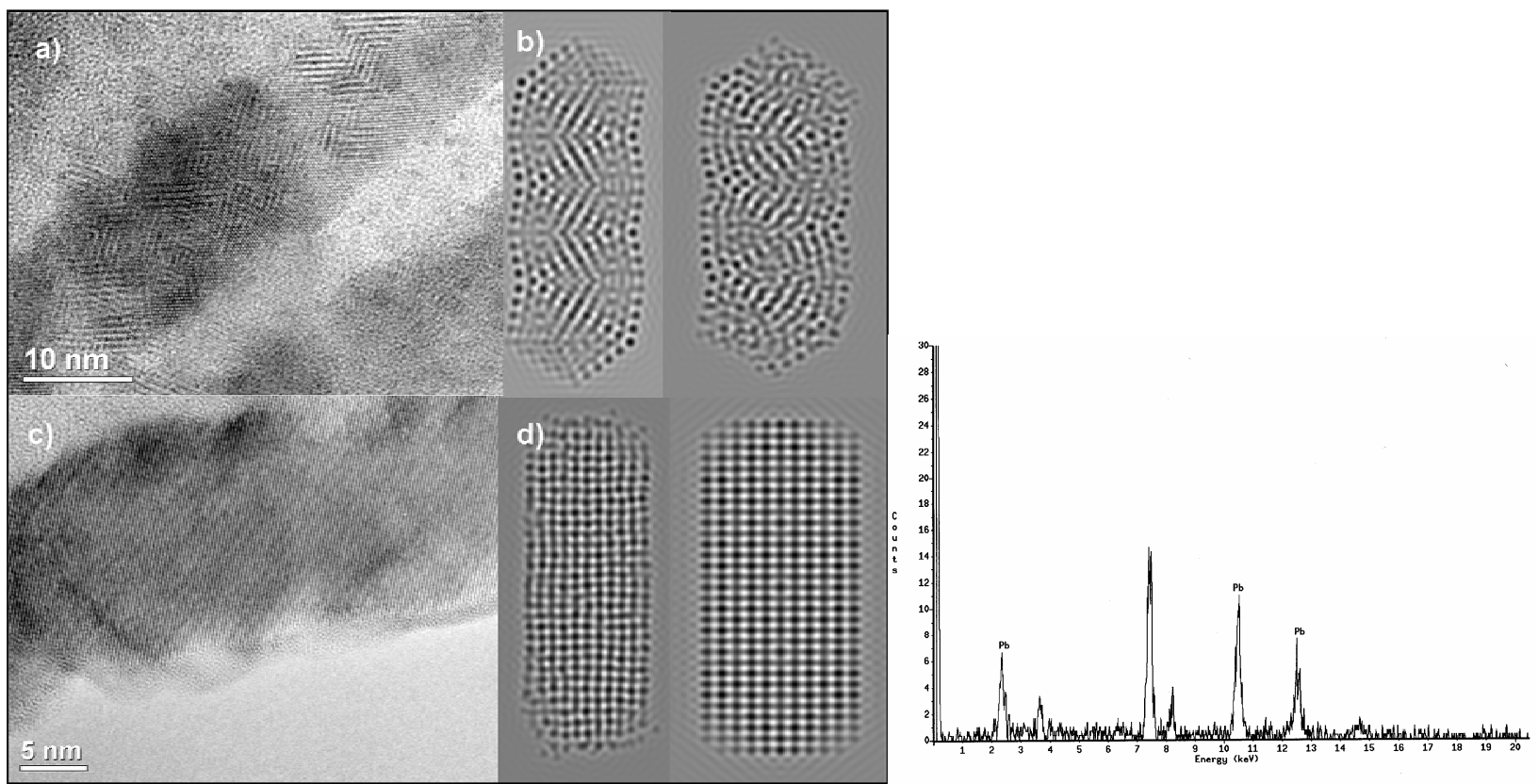

Figure 3. a) HRTEM micrograph of single Pb nanowire showing multiple twining planes (MTP).

b) HRTEM theoretical images obtained from Simulated Annealing algorithms, the initial stages of the annealing shows the presence of MTP which is in good agreement with the experimental results. c) HRTEM images of $\mathrm{Pb}$ monocrystal wire. d) HRTEM theoretical images show the evolution of the wires up to a final stage which corresponds to a monocrystaline wire. e) EDS microanalysis obtained in nanobeam mode. No oxygen is present. 